\title{
Biocompuestos de Harina de Yuca obtenidos por Termo- Compresión. Efecto de las Condiciones de Proceso
}

\author{
Diana P. Navia $a^{(1)}$, Alfredo A. Ayala ${ }^{(2)}$ y Héctor S. Villada ${ }^{(3)}$ \\ (1) Universidad de San Buenaventura Seccional Cali, Facultad de Ingeniería, Programa de Ingeniería \\ Agroindustrial, Avenida 10 de mayo, La Umbría, Vía a Pance, Cali, Valle del Cauca-Colombia. \\ (e-mail: dpnavia@usbcali.edu.co). \\ (2) Universidad del Valle, Escuela de Ingeniería de Alimentos, Ciudad Universitaria Meléndez, \\ A.A. 25360 Cali-Colombia. (e-mail: alfredo.ayala@correounivalle.edu.co) \\ (3) Universidad del Cauca, Facultad de Ciencias Agrarias, Departamento de Agroindustria, \\ Calle 5 \# 4-70, Popayán-Colombia. (e-mail: villada@unicauca.edu.co)
}

Recibido Mar. 24, 2015; Aceptado May. 29, 2015; Versión final Jul. 6, 2015, Publicado Oct. 2015

\begin{abstract}
Resumen
El objetivo de este estudio fue evaluar el efecto de las condiciones del proceso de obtención de biocompuestos elaborados por la técnica de termo-compresión usando harina de yuca de variedad MPER 183, fibra de fique y glicerol. Se ha analizado contenido de agente de expansión, presión de compresión y humedad relativa sobre la densidad, esfuerzo de flexión y adsorción de vapor de agua. Los resultados mostraron que todas las condiciones de procesamiento evaluadas afectaron significativamente las variables de respuesta, con un nivel de significancia del $95 \%(p<0,05)$. El aumento del bicarbonato de sodio como agente de expansión y los valores altos de humedad relativa incrementaron la densidad, adsorción de vapor agua y disminuyeron el esfuerzo de flexión. La ausencia de presión durante la compresión aumentó el esfuerzo de flexión, y disminuyó la densidad y la adsorción de vapor de agua de los biocompuestos.
\end{abstract}

Palabras clave: densidad; esfuerzo; flexión; bioplásticos; yuca

\section{Cassava Flour Biocomposites made by Thermopressing. Effect of Processing Conditions}

\begin{abstract}
The objective of this study was to evaluate the effect of process conditions on the production of biocomposites prepared by thermopressing technique, using cassava flour variety MPER 183, fique fiber and glycerol. The variables analyzed were: content of blowing agent, compression pressure and relative humidity on density, flexion stretch, and water vapor adsorption. The results showed that all processing conditions evaluated had significant effect $(p<0,05)$ on response variables. When both sodium bicarbonate as blowing agent and the values of relative humidity were increased, the density and water vapor adsorption values also increased, while flexion stretch values decreased. The absence of pressure during the compression increased the flexion stretch values, and decreased density and the water vapor adsorption values of biocomposites.
\end{abstract}

Keywords: density; stress; flexion; bioplastics; cassava 


\section{INTRODUCCIÓN}

Los materiales plásticos se han utilizado desde hace más de cien años en diversas aplicaciones especialmente en el sector de empaques, debido a que presentan características deseables tales como estabilidad estructural, resistencia a las agresiones del medio, bajo costo, tecnologías de escala y metodologías de producción altamente tecnificadas, entre otros beneficios (Zárate-Ramírez et al., 2014). Sin embargo, la alta demanda de estos productos (280 millones de toneladas en 2011) y su rápida disposición final después de su uso (Luijsterburg y Goossens, 2014), han llevado a una acumulación de estos materiales petroquímicos en el medio ambiente generando altos niveles de contaminación. Lo anterior, debido a la baja degradación de este tipo de plásticos, lo que ocasiona serios problemas ambientales en razón a los largos periodos de descomposición, algunos hasta de 500 años o más.

A nivel global se han encaminado investigaciones por la obtención de plásticos biodegradables, desarrollándose numerosos estudios alrededor de esta temática, por cuanto encontrar alternativas viables que sustituyan los polímeros convencionales derivados del petróleo se ha convertido en una necesidad. Es así, como mundialmente, los bioplásticos representan la línea de productos de mayor crecimiento en la industria de productos de base biológica (Morone et al., 2015).

De acuerdo con lles y Martin (2013), los bioplásticos pueden unir productores de materias primas (recursos naturales renovables) y comercializadores, a través del desarrollo de nuevas tecnologías para la producción de prototipos que cumplan con los requerimientos comerciales de durabilidad, rendimiento y costos. "MaterBi" es un producto desarrollado por "Novamont" a partir de almidón y celulosa, con el cual se producen y comercializan actualmente empaques biodegradables. Estos empaques elaborados a partir de recursos renovables provenientes de plantas y/o animales, ofrecen una solución conveniente y amigable con el ambiente, considerándose como estrategias para reducir las emisiones de dióxido de carbono y la dependencia de recursos fósiles (Peelman et al., 2013). Por lo anterior, es importante investigar los efectos de las técnicas de procesamiento y materias primas con las que se obtienen los bioplásticos, en orden de identificar sus potencialidades y limitaciones.

Dentro de los recursos renovables que pueden ser utilizados en Colombia en la elaboración de materiales biodegradables, se encuentran las materias primas ricas en almidón, principalmente raíces y tubérculos debido a su disponibilidad, ya que tienen un área representativa de cultivo en el país. Las materias primas principales usadas para la obtención del bioplástico "yuca" y "fique", son productos de amplia disponibilidad y bajo costo en Colombia, con gran potencial en el aprovechamiento agroindustrial, además de sus bondades con el proceso de termo-plastificación para la obtención del bioplástico y su calidad renovable y biodegradable.

Por su parte, la técnica de termo-compresión usada para la obtención del material implica costos de producción relativamente bajos, aspecto clave para que la tecnología pueda ser transferida y aplicada en mayor escala en etapas futuras de la investigación, además, permite obtener el producto con las características de resistencia y rigidez requeridas. El objetivo de esta investigación fue estudiar las condiciones de proceso en la producción de un empaque semirrígido, evaluando su densidad y resistencia mecánica en términos de esfuerzo de flexión.

\section{MATERIALES Y MÉTODOS}

Se describen las materias primas usadas y su preparación, se explica el cálculo de la densidad, se explica la determinación de las propiedades mecánicas y de la adsorción de vapor de agua, terminando por detallar el diseño experimental usado.

\section{Materias primas y preparación}

Se prepararon suspensiones con 6 partes de agua $(60 \mathrm{~g})$ por cada 4 de harina $(40 \mathrm{~g})$ de la variedad de yuca MPER 183. Las suspensiones de harina se calentaron a $75^{\circ} \mathrm{C}$ con agitación de $125 \mathrm{rpm}$ hasta su gelatinización. Posteriormente se adiciono fibra de fique con 10\% de humedad y tamaño medio de partícula de $250 \mu \mathrm{m}$, glicerol USP con pureza de $99.00 \%$ (Sigma-Aldrich $\AA$ ) como agente plastificante, de acuerdo a la siguiente formulación con respecto al peso total: $60 \%$ harina gelatinizada, $25 \%$ fibra y $15 \%$ plastificante. Simultáneamente, se incorporó bicarbonato de sodio como agente de expansión en proporciones de 0 a $11 \%$ del peso total de la formulación, según diseño experimental de la tabla 1. Finalmente, las muestras fueron obtenidas por termo-compresión durante $3 \mathrm{~min}$ a $200^{\circ} \mathrm{C}$, en una prensa hidráulica (Edafa $\Theta$, Colombia), depositando la mezcla en moldes de teflón de $25 \mathrm{~cm}$ de largo y $20 \mathrm{~cm}$ de ancho. Las dimensiones de las muestras para flexión fueron $12.7 \mathrm{~cm}$ de largo, $1.27 \mathrm{~cm}$ de ancho y $3 \mathrm{~mm}$ de espesor, según norma ASTM D790-10. La humedad de las materias primas se determinó por desecación en estufa, según método AOAC 934. 


\section{Densidad}

La densidad fue calculada como la relación entre el peso y el volumen de las muestras. Los valores reportados corresponden al promedio de 10 mediciones de cada uno de los 27 tratamientos descritos en el diseño experimental de la tabla 1.

\section{Propiedades mecánicas}

La resistencia a la flexión se evaluó bajo las especificaciones de la norma ASTM D790. Se determinó el esfuerzo en la rotura $\left(\sigma_{F}\right)$ en muestras acondicionadas durante diez días a $23 \pm 1^{\circ} \mathrm{C}$ y 70,80 y $90 \%$ de humedad relativa $(H R)$. Se evaluaron 7 muestras por tratamiento. Se utilizó un texturómetro (Shimadzu, EZ Test L, Japón), equipado con una celda de carga de $500 \mathrm{~N}$. El ( $\left.\sigma_{F}\right)$ fue obtenido de la fracción entre la fuerza máxima y el área transversal de la muestra, mediante el software Trapezium (versión 2.33) acoplado al texturómetro.

\section{Adsorción de vapor de agua}

El contenido de humedad en equilibrio (CHE) de las bandejas fue calculado mediante procedimientos de adsorción de vapor de agua. Las muestras de $2 \mathrm{~cm} \times 2 \mathrm{~cm}$ del material $(0.8 \pm 0.1$ gramos) fueron colocadas en recipientes herméticos con soluciones salinas saturadas a 70, 80 y 90\% de humedad relativa (HR). Estos recipientes se ubicaron en una cámara ambiental (KBF115 Binder $\AA$, USA) a temperatura constante de $25^{\circ} \mathrm{C}$, durante 30 días, al cabo de los cuales, por gravimetría (diferencia entre peso final e inicial de la muestra), se determinó el $\mathrm{CHE}$ de las mismas. Se emplearon las sales $\mathrm{NaCl}, \mathrm{KCl}$, y $\mathrm{BaCL} 2$ cuya saturación genero valores de HR de 70,80 y $90 \%$ respectivamente.

\section{Diseño experimental}

La tabla 1 describe la codificación de los factores, los cuales combinados con los niveles respectivos, resultan en 27 tratamientos. Se empleó el software SPSS versión 19 para someter los resultados del diseño experimental a prueba de normalidad ("Kdmogorov-Smirnov" para datos mayores e iguales a 50 y "ShapiroWilk" para datos menores a 50).

Tabla 1: Codificación de los factores y niveles del diseño experimental rotatorio

\begin{tabular}{|c|c|c|c|c|}
\hline Factores & Código & \multicolumn{2}{|c|}{ Niveles } & Variables de Respuesta \\
\hline \multirow{3}{*}{$\begin{array}{l}\text { Presión de compresión } \\
\text { (MPa) }\end{array}$} & \multirow{3}{*}{$\mathrm{X} 1$} & 0 & Bajo (-1) & \multirow{9}{*}{$\begin{array}{l}\text { Densidad } \\
\text { Esfuerzo de Flexión } \\
\text { Adsorción de vapor de agua }\end{array}$} \\
\hline & & 0,6 & Centro (0) & \\
\hline & & 1,2 & Alto (1) & \\
\hline \multirow{3}{*}{ Agente de expansión (\%) } & \multirow{3}{*}{$\mathrm{X} 2$} & 0 & Bajo $(-1)$ & \\
\hline & & 5,5 & Centro (0) & \\
\hline & & 11 & Alto (1) & \\
\hline \multirow{3}{*}{$\begin{array}{l}\text { Humedad relativa de } \\
\text { almacenamiento (\%) }\end{array}$} & \multirow{3}{*}{ X3 } & 70 & Bajo (-1) & \\
\hline & & 80 & Centro (0) & \\
\hline & & 90 & Alto (1) & \\
\hline
\end{tabular}

Los datos resultantes "normales" se evaluaron a través de análisis de varianza con nivel de confianza del $95 \%$, previa prueba de Levene (homogeneidad de varianzas) y finalmente análisis post-varianza para los datos estadísticamente significativos, a través de la prueba de "Tukey" para varianzas homogéneas o "T3 Dunnett" para varianzas no homogéneas. Adicionalmente, se empleó el análisis por superficies de respuesta usando el software Matlab (2008a), con el cual se identificaron los modelos matemáticos correspondientes a los experimentos desarrollados y se obtuvieron las gráficas de superficie de respuesta tridimensionales con los gráficos de contorno respectivos.

\section{RESULTADOS Y DISCUSIÓN}

\section{Densidad}

Los resultados del análisis estadístico, determinaron que los factores "presión de compresión", "humedad relativa" y contenido de "agente de expansión" afectaron significativamente los resultados de densidad de los bioplásticos $(p<0,05)$, tanto independientemente como en interacción o combinación de factores. El análisis de varianza estableció que la presión fue el factor de mayor efecto sobre la densidad. Las superficies de respuesta de la figura 1, muestran la variación de la densidad, en condiciones fijas de humedad relativa y presión de compresión, en bioplásticos elaborados con harina de yuca de variedad MPER 183. 
Los valores bajos de densidad son una característica deseada en los bioplásticos obtenidos, debido a la disminución de costos, al utilizar menor cantidad de material, además, un producto con menor peso favorece sus condiciones de comercialización. El menor valor obtenido para este parámetro físico fue $0,39 \mathrm{~g} / \mathrm{cm}^{3}$ (MPER 183) y 0,40 g/cm³ (CM 4574-7), ambos, con $0 \%$ de agente de expansión y 0 MPa de presión en la compresión bajo un ambiente con $70 \%$ de humedad relativa. En la figura 1, puede notarse que al incrementar la presión, los valores de densidad de las muestras aumentan. Este fenómeno, obedece a que los valores altos de presión en el proceso de compresión obstaculizan la salida del vapor de agua desde el interior de la muestra, y consecuentemente, se limita la formación de poros y espacios intersticiales en la estructura del bioplástico, los cuales promueven la expansión de la estructura del material, por lo tanto, el aumento en la presión de compresión no favoreció la densidad del bioplástico. Este fenómeno fue reportado por Han y Yoo (1981), quienes afirmaron, que las presiones bajas favorecieron la formación de burbujas en los productos moldeados obtenidos, mientras que las altas no.
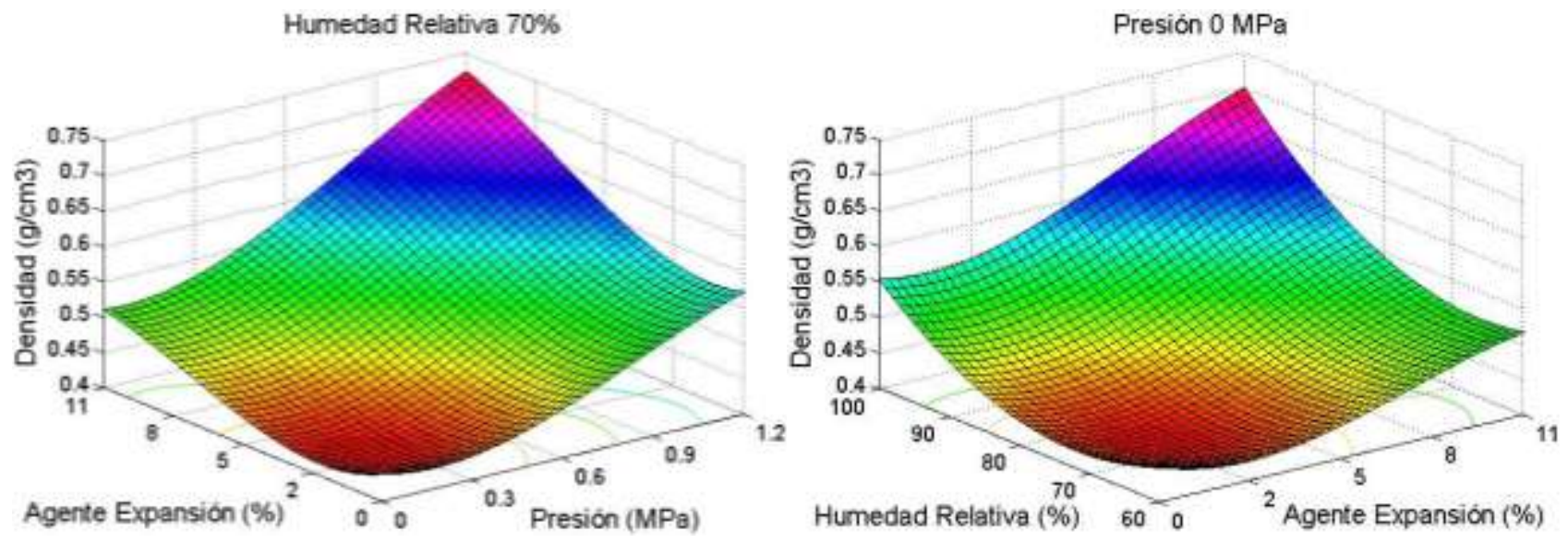

Fig. 1: Superficies de respuesta para densidad de los biocompuestos

Por su parte, la densidad aumentó con el incremento del contenido de agente de expansión en la matriz termoplástica. Este fenómeno podría explicarse, por la presencia insuficiente de agua para producir el gas a partir de la descomposición térmica del bicarbonato de sodio. El dióxido de carbono (gas), es producido a partir del bicarbonato de sodio, según la siguiente reacción (Berrios et al., 2004):

$2 \mathrm{NaHCO}_{3}----$ Calor $+\mathrm{H} 2 \mathrm{O} \rightarrow \mathrm{Na}_{2} \mathrm{CO}_{3}+\mathrm{CO}_{2}+\mathrm{H}_{2} \mathrm{O}$

La temperatura del inicio de la descomposición del bicarbonato de sodio es $87,7^{\circ} \mathrm{C}$ (Thieme et al., 2012), por lo que éste se ha usado en procesos de termo-compresión entre 140 y $200^{\circ} \mathrm{C}$ para generar expansión en las matrices termoplásticas (Najib et al., 2011; Tanrattanakul y Chumeka, 2010). Sin embargo, Zhou y Hanna (2004) afirmaron que a pesar del incremento en el contenido de bicarbonato de sodio, la expansión de las muestras no aumentó por causa del contenido bajo de agua, mientras que cuando la proporción del líquido aumentó, la formación de gas también y la densidad de las muestras se redujo por causa de la expansión del material. De forma similar, Tanrattanakul y Chumeka, 2010, elaboraron espumados de almidón por termocompresión a $180^{\circ} \mathrm{C}, 5 \mathrm{~min}$ y 1000 psi, y reportaron que el incremento en el contenido de bicarbonato de sodio no presentó variaciones en la densidad del material.

Por su parte, la densidad incrementó con el aumento de la humedad relativa de almacenamiento. Este comportamiento puede explicarse por la ganancia en peso adquirida por las muestras por efecto de la adsorción de agua en la superficie de las mismas, ya que su carácter es predominantemente hidrófilo, por tanto, cuanto más alto sea el valor de humedad en el medio en que se encuentran, mayor será el vapor de agua adherido a su superficie, aumentando por consiguiente, su densidad.

De otro lado, se han reportado valores de densidad, en materiales espumados obtenidos por termocompresión a partir de almidón y fibras de caña de azúcar entre 0,19 y 0,29 g/cm³ (Vercelheze et al., 2012), en bandejas biodegradables obtenidas por termo-compresión a partir de almidón, reforzadas con fibras de celulosa entre 0,45 y 0,68 $\mathrm{g} / \mathrm{cm}^{3}$ (Salgado et al., 2008), bandejas termo-comprimidas obtenidas a partir de almidón de yuca, quitosano y fibras naturales con valores entre 0,12 y 0,15 g/cm3 (Kaisangsri et al., 2012) y bandejas fabricadas a partir de almidón de yuca, con proteína de maíz, soya y trigo y fibras de kenaf, obtenidas por termo-compresión entre 0,2 y $0,8 \mathrm{~g} / \mathrm{cm} 3$ (Kaisangsri et al., 2014). Si bien, la densidad de los bioplásticos es similar a la reportada en otros materiales obtenidos por termo-compresión, ésta, es aún muy alta comparada con la del poliestireno expandido que está entre 0,05 y 0,09 g/cm ${ }^{3}$ (Kaisangsri et al., 2014), que es el polímero fósil objetivo de sustitución con los materiales obtenidos en este estudio. 


\section{Flexión}

Los resultados del análisis estadístico, determinaron que los factores "presión de compresión", "humedad relativa" y contenido de "agente de expansión" afectaron significativamente los resultados de esfuerzo de flexión $\left(\sigma_{F}\right)(p<0,05)$. Las superficies de respuesta y contornos de la figura 2,3 y 4 , muestran la variación de $\sigma_{F}$ en condiciones fijas de humedad relativa y contenido de agente de expansión, respectivamente, en los bioplásticos. En la figura 3, al aumentar la humedad relativa, se nota que el esfuerzo de flexión disminuye en los bioplásticos, en razón a que, el incremento del porcentaje de humedad relativa representa un incremento de vapor de agua disponible en el medio, el cual, por su afinidad con los componentes del bioplástico, favorece la pérdida de propiedades de resistencia al aumentar la movilidad molecular al interior de la estructura. De acuerdo con lo anterior, Mello y Mali (2014) quienes fabricaron bandejas espumadas basadas en almidón de yuca, reportaron que la hidrofilicidad de las moléculas de almidón favorece la formación de puentes de hidrogeno con el agua. El agua actúa como plastificante, reduciendo las interacciones y la proximidad entre las cadenas poliméricas (amilosa/amilopectina) del almidón, incrementando el volumen libre entre dichas moléculas, de manera que bajo esfuerzos mecánicos, los movimientos de las cadenas del almidón se facilitan y esto se refleja en la disminución del esfuerzo del material. Por otra parte, Lee et al., (2013) reportaron que el incremento de la disponibilidad de agua disminuyó la resistencia mecánica de láminas bioplásticas termocomprimidas, debido a que el agua ganada actúa como plastificante, aumentando la flexibilidad de las muestras y disminuyendo consecuentemente su esfuerzo mecánico.
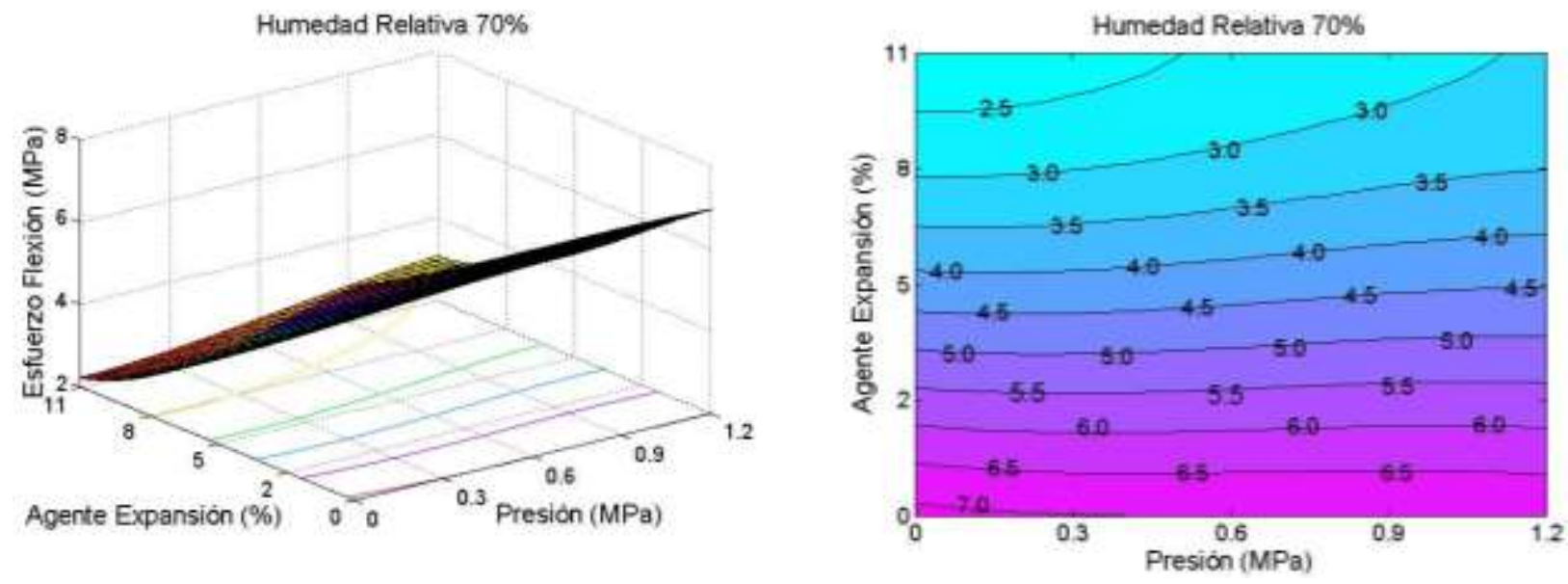

Fig. 2: Superficie de respuesta y contorno para esfuerzo de flexión: humedad relativa fija
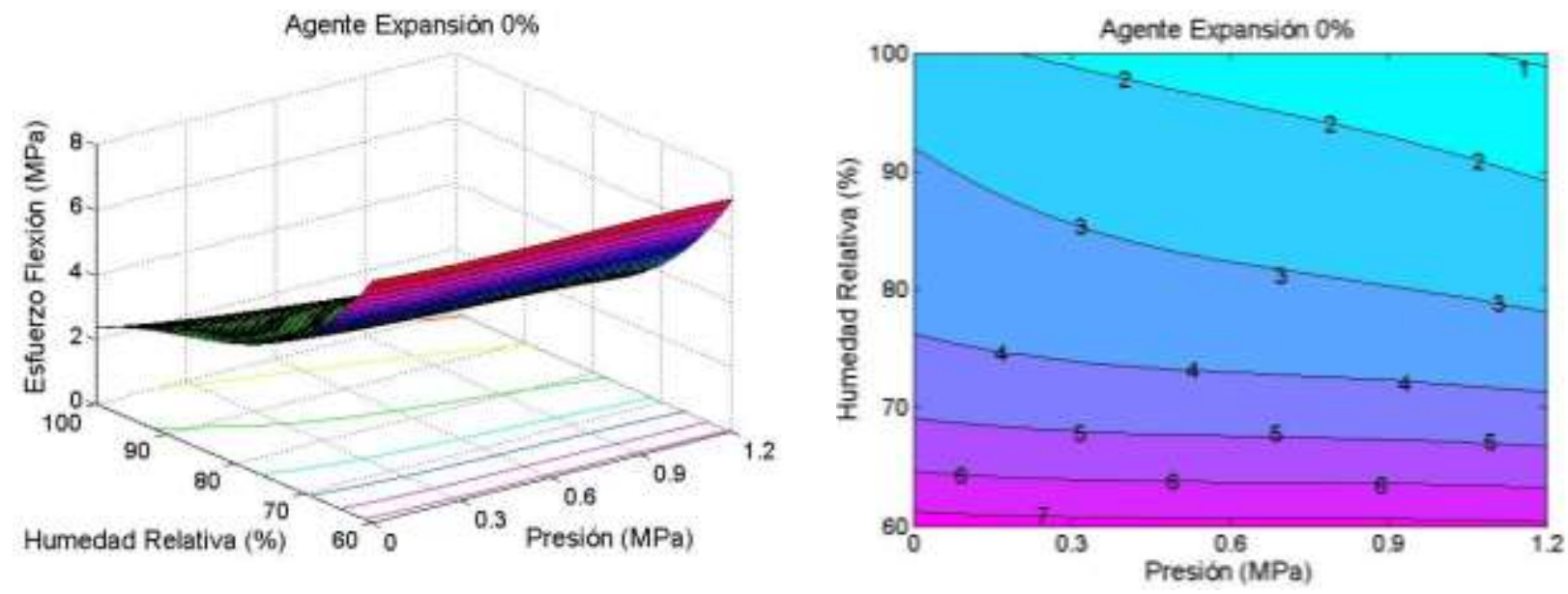

Fig. 3: Superficie de respuesta y contorno para esfuerzo de flexión: agente expansión fijo

De otro lado, el efecto de la presión evidenciado en las figuras 2 y 3 , indica que su aumento no favorece el esfuerzo de flexión. Este comportamiento, se relaciona posiblemente con un cambio estructural de la matriz termoplástica ocasionado por la acción combinada de calor y alta presión a la que fue sometida en la termocompresión, en donde posiblemente se generaron aglomerados de amilosa/amilopectina con fracciones proteicas y de fibra desnaturalizadas. Estas aglomeraciones, desfavorecen la plastificación uniforme del 
biopolímero, ya que concentran la capacidad de resistencia mecánica en algunos puntos del material, debilitando las zonas restantes, ocasionando que su respuesta ante los esfuerzos mecánicos disminuya.

Al respecto, algunos investigadores han argumentado que las técnicas de procesamiento que involucran calor y presión, generan modificaciones en la estructura del material, como disociación de las macromoléculas poliméricas, pero a la vez, pueden formarse acumulaciones en puntos específicos de la matriz termoplastificada, que no favorecen su desempeño mecánico. En este sentido, se ha reportado que en materiales amiláceos, las altas temperaturas de procesamiento pueden desnaturalizar los componentes de la matriz, los cuales forman aglomeraciones con el almidón gelatinizado, minimizando los esfuerzos mecánicos (González et al., 2011). Entre tanto, el incremento del agente de expansión promueve la disminución de las propiedades mecánicas de los bioplásticos, como se evidencia en la figura 2, donde al aumentar el contenido de agente de expansión, los valores de esfuerzo de flexión disminuyen. Resultados similares, fueron reportados por Soares y Bohrz 2013 y Lee et al., 2009.

A pesar de que el agente de expansión usado, no generó disminución en la densidad como era lo esperado, es posible que su descomposición promueva la ganancia de agua de los bioplásticos, debido a los productos residuales que se generan, se solubilizan y permanecen en la matriz termoplástica, reduciendo así las propiedades mecánicas de flexión. De acuerdo con Muñoz (2014), la descomposición del bicarbonato de sodio, genera productos residuales que permanecen en el polímero y que no benefician la expansión. Es posible también, que el tiempo de termo-compresión $(5 \mathrm{~min}$ ) con el que fueron obtenidos los bioplásticos haya sido insuficiente para la descomposición completa del agente de expansión y la consecuente formación de gas. Por lo anterior, gran parte del bicarbonato de sodio podría permanecer en la matriz y dado su solubilidad en agua, se facilita la ganancia la misma, conforme aumenta el porcentaje de humedad relativa de almacenamiento, y así, se disminuye el esfuerzo de flexión. En este contexto, Muñoz (2014), afirmó que la producción de gas usando bicarbonato de sodio como agente de expansión, ocurrió a los 16 min a temperatura de $180^{\circ} \mathrm{C}$.

El comportamiento mecánico promedio de los bioplásticos elaborados con fue de $8.8 \mathrm{MPa}(\sigma \mathrm{F})$. Los valores encontrados en estas propiedades fueron superiores a los reportados por Uslu y Polan (2012), en espumados de almidón de maíz $3.56 \pm 1.3 \mathrm{MPa}\left(\sigma_{\mathrm{F}}\right)$, con densidad de $0.4 \mathrm{~g} / \mathrm{cm}^{3}$; y Kaisangsri et al., 2014, quienes estudiaron espumados de almidón de yuca $4.18 \mathrm{MPa}\left(\sigma_{\mathrm{F}}\right)$ y densidad de $0.46 \mathrm{~g} / \mathrm{cm}^{3}$. En general, las muestras de bioplásticos presentaron valores superiores a los reportados en poliestireno expandido con $\sigma_{\mathrm{F}}: 0.96 \mathrm{MPa}$ (Navia et al., 2013), por lo cual se consideran técnicamente factibles en cuanto a propiedades mecánicas como alternativa de uso para empacar productos alimentarios.

\section{Adsorción de vapor de agua}

Los resultados del análisis estadístico, determinaron que los factores "presión de compresión", "humedad relativa" y contenido de "agente de expansión" afectaron significativamente los resultados de adsorción de vapor de agua, expresado en términos de contenido de humedad de equilibrio ( $\mathrm{CHE}$ ) de los bioplásticos $(p<0.05)$. Las superficies de respuesta y contornos de la figura 4 muestran la variación en el CHE, en condiciones fijas de humedad relativa en los biocompuestos. Cuando el porcentaje de humedad relativa de almacenamiento aumenta, consecuentemente hay mayor disponibilidad de agua en el medio y el valor de CHE aumenta. Esto se debe, al carácter hidrófilo de la matriz termoplástica, cuyos componentes principales (harina, fibra, glicerol) son afines al agua.

En la figuras 4, puede notarse la influencia del contenido de bicarbonato de sodio (agente de expansión) sobre el CHE. El aumento desde 0 a $8 \%$ en el contenido de bicarbonato de sodio incrementa los valores de $\mathrm{CHE}$ desde 5 hasta $15 \%$ b.s., y posteriormente, se presenta una disminución en CHE hasta $11 \%$ b.s., este último valor cuando el contenido de bicarbonato de sodio aumenta hasta $11 \%$.De forma análoga, en humedades relativas del 80 y $90 \%$ (resultados no mostrados), se presentó el mismo comportamiento.

Este fenómeno, posiblemente es consecuencia de la descomposición incompleta del bicarbonato de sodio, a causa del corto tiempo durante la termo-compresión (5 min). Muñoz (2014), estudió la formación de gas a partir de la descomposición del bicarbonato de sodio y afirmó que éste, se produjo al minuto 16 a una temperatura de $180^{\circ} \mathrm{C}$. En razón a lo anterior, probablemente, la sal residual que no alcanzó su descomposición durante el procesamiento del bioplástico, podría solubilizarse y generar puntos activos que incrementan la ganancia de vapor de agua y con ello, un aumento en el valor de CHE. De forma similar, es posible que algunos residuos, que se generan de la descomposición de la sal, permanezcan en la matriz termoplástica favoreciendo el aumento de CHE con su solubilización. 

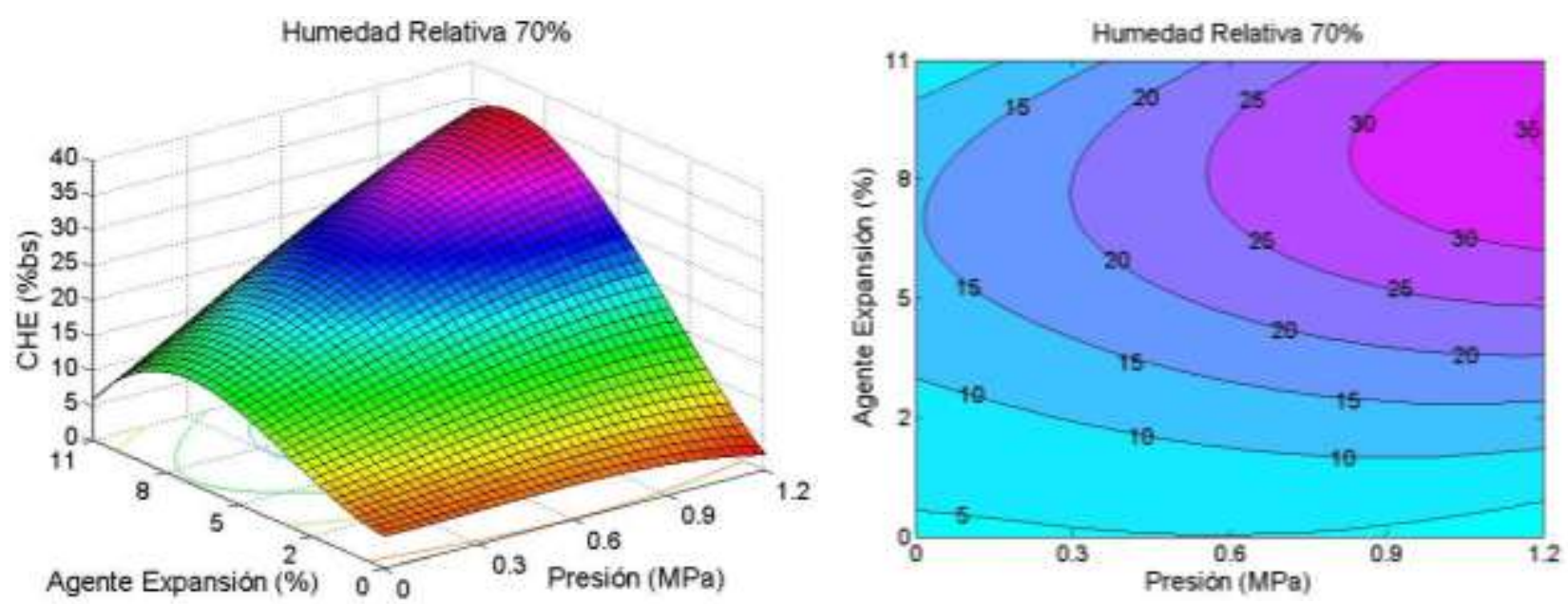

Fig. 4: Superficie de respuesta y contorno para CHE de los biocompuestos

Por su parte, el incremento inicial en el CHE y posterior descenso, presentado con el aumento del contenido de bicarbonato de sodio en valores constantes de presión, podría relacionarse con un fenómeno de saturación de la sal alcanzado en $8 \pm 0,5 \%$. Probablemente, después de este valor, la sal se sobresatura promoviendo una competencia por los sitios activos de adsorción de agua, entre la sal (oxígeno de los grupos carbonilo) y el agua del medio de almacenamiento para formar enlaces con los grupos hidroxilo (almidón y glicerol) en la matriz termoplástica; y por tanto, esta competencia, ocasione la disminución en los valores de CHE después de la saturación de la sal.

Entre tanto, los valores altos de presión promueven altos valores de $\mathrm{CHE}$, conforme aumenta la concentración de agente de expansión. Una mayor presión, implica mayor hermeticidad entre los moldes durante la compresión, por lo cual, se dificulta el proceso de formación de gas y salida del mismo para la expansión. Es posible, que a valores altos de presión, se obstaculice la descomposición del bicarbonato de sodio, resultando en cantidades remanentes de sal disponibles para solubilizarse con la humedad del medio, generando posiblemente mayor número de puntos activos, y en consecuencia, las muestras adsorben más humedad hasta la concentración de saturación de la sal $(8 \pm 0,5 \%)$. En efecto, se evidencio una región óptima en las figura 4, representada con color rosado, la cual indica un valor máximo alcanzado de $\mathrm{CHE}$ en condiciones de alta presión y $8 \pm 0,5 \%$ de agente de expansión. Estos valores máximos fueron $35 \%$ b.s. en $70 \%$ de humedad relativa, mientras que en $90 \%$ de humedad relativa fue $45 \%$ b.s.

\section{CONCLUSIONES}

Se evidenció un efecto significativo $(p<0,05)$ de los factores "presión de compresión", "humedad relativa" y contenido de "agente de expansión" sobre la densidad, propiedades mecánicas y adsorción de vapor de agua en los bioplásticos. Los resultados de densidad, esfuerzo de flexión y contenido de humedad en equilibrio, indicaron que las condiciones más favorables para la obtención de los bioplásticos por termo-compresión con harina de variedad de yuca MPER 183, fueron 0 MPa de presión y $0 \%$ de contenido de bicarbonato de sodio. Bajo estas condiciones, es posible obtener biocompuestos para evaluar futuras aplicaciones en el campo alimentario y no alimentario.

Las investigaciones futuras deben orientarse a la reducción de los valores de CHE y densidad del bioplástico, para establecer oportunidades de mercado que permitan la sustitución parcial/total del poliestireno.

\section{AGRADECIMIENTOS}

Se resalta el apoyo de la Universidad del Cauca y Universidad de San Buenaventura Seccional Cali.

\section{REFERENCIAS}

Berrios, J., y otros cuatro autores, Sodium bicarbonate and the microstructure, expansion and color of extruded black beans, Journal of Food Processing and Preservation 28, 321-335 (2004)

González, J., y otros tres autores, Effect of processing on the viscoelastic, tensile and optical properties of albumen/starch-based bioplastics, Carbohydrate Polymers, 84 (1), 308-315 (2011) 
Han, C., y Yoo, H., Studies on Structural Foam Processing. IV. Bubble Growth During Mold Filling, Polymer Engineering and Science, 21(9), 518-533 (1981)

lles, A., y Martin, D., Expanding bioplastics production: sustainable business innovation in the chemical industry, Journal of Cleaner Production 45(), 38-49 (2013)

Kaisangsri, N., Kerdchoechuen, O., y Laohakunjit, N., Biodegradable foam tray from cassava starch blended with natural fiber and chitosan, Industrial Crops and Products, 37 (1), 542- 546 (2012)

Kaisangsri, N., Kerdchoechuen, O., y Laohakunjit, N., Characterization of cassava starch based foam blended with plant proteins, kraft fiber, and palm oil, Carbohydrate Polymers, 110 (1), 70-77 (2014)

Lee, R., y otros tres autores, Influence of glycerol and water activity on the properties of compressed egg white-based bioplastics, Journal of Food Engineering, 118(1), 132-140 (2013)

Lee, S., y otros tres autores, Evaluation of ingredient effects on extruded starch-based foams using a supersaturated split-plot design, Industrial Crops and Products 29 (2-3), 427-436 (2009)

Luijsterburg, B., y Goossens, H., Assessment of plastic packaging waste: Material origin, methods, properties, Resources, Conservation and Recycling 85(1) 88-97 (2014)

Mello, L., y Mali, S., Use of malt bagasse to produce biodegradable baked foams made from cassava starch, Industrial Crops and Products, 55(1), 187-193 (2014)

Morone, P., Tartiu, V., y Falcone, P., Assessing the potential of biowaste for bioplastics production through social network analysis, Journal of Cleaner Production 90(1), 43-54 (2015)

Muñoz, S., Desarrollo de nuevos agentes espumantes endotérmicos para la fabricación de materiales celulares poliméricos, Tesis de grado de Física. Universidad de Valladolid, Facultad de Ciencias, España: Valladolid, pp. 83 (2014)

Najib, N., y otros tres autores, Correlation between the acoustic and dynamic mechanical properties of natural rubber foam: Effect of foaming temperature, Materials and Design 32 (2), 505-511 (2011)

Navia, D., Villada, H., y Ayala, A., Evaluación mecánica de bioplásticos semirrígidos elaborados con harina de yuca, Biotecnología en el sector Agropecuario y Agroindustrial, Ed Especial 2, 77-84 (2013)

Peelman, N., y otros seis autores, Application of bioplastics for food packaging, Trends in Food Science \& Technology 32(2), 128-141 (2013)

Salgado, P., y otros cuatro autores, Biodegradable foams based on cassava starch, sunflower proteins and cellulose fibers obtained by a baking process, Journal of Food Engineering 85 (3), 435-443 (2008)

Soares, F., y Bohrz, S., Effect of chemical and physical foaming additives on the properties of PP/wood flour composites, Polymer Testing, 32 (4), 640-646 (2013)

Tanrattanakul, V., y Chumeka, W., Effect of potassium persulfate on graft copolymerization and mechanical properties of cassava starch/natural rubber foams, Journal of Applied Polymer Science 116 (1), 93-105 (2010)

Thieme, C., Sodium Carbonates in Ullman's Encycopedia of Industrial Chemistry by Solvay Alkali GmbH, pp 299-316, Wiley, Federal Republic of Germany, Weinheim, (2012)

Tunjano, V., y otros cinco autores, Estudio de las propiedades térmicas y mecánicas del almidón termoplástico (TPS) reforzado con nanoarcilla, Revista latinoamericana de metalurgia y materiales. S1(1), 29-36 (2009)

Uslu, M., y Polat, S., Effects of glyoxal cross-linking on baked starch foam, Carbohydrate Polymers 87 (3), 1994- 1999 (2012)

Vercelheze, A., y otros seis autores, Properties of baked foams based on cassava starch, sugarcane bagasse fibers and montmorillonite, Carbohydrate Polymers, 87 (2), 1302-1310 (2012)

Zárate-Ramírez, L., y otros cuatro autores, Thermo-mechanical and hydrophilic properties of polysaccharide/gluten-based bioplastics, Carbohydrate Polymers 112(1), 24-31 (2014)

Zhou, J., y Hanna, M., Extrusion of starch acetate with mixed blowing agents, Starch/Stärke 56 (10), 484-494 (2004) 